\title{
Regression of ventriculomegaly following medical management of a patient with Hurler syndrome
}

\author{
Jennifer Liang, BHK, MSc, PT, and Ash Singhal, MD, FRCSC \\ Faculty of Medicine and the Division of Neurosurgery, University of British Columbia and British Columbia's Children's Hospital, \\ Vancouver, British Columbia, Canada
}

\begin{abstract}
Hurler syndrome is the most severe form of mucopolysaccharidosis (MPS) Type 1. Progressive neurocognitive decline in this condition can be accompanied by macrocephaly, ventriculomegaly, and/or periventricular signal changes on MRI, which often leads to a neurosurgical referral. In this case, the authors describe a 2-year-old boy with ventriculomegaly and periventricular T2 signal changes, both of which decreased following medical management of Hurler syndrome. The authors discuss the possible mechanisms for this finding and the implications for neurosurgical treatment of this condition.
\end{abstract}

http://thejns.org/doi/abs/10.3171/2015.9.PEDS15477

KEY WORDS mucopolysaccharidosis Type 1; Hurler syndrome; ventriculomegaly; pediatric neurosurgery; congenital

$\mathrm{M}$ UCOPOLYSACCHARIDOSIS (MPS) Type 1 is an autosomal-recessive lysosomal storage disorder affecting approximately 1 in 100,000 births..$^{10}$ Affected children have a deficiency in the lysosomal enzyme $\alpha$-1-iduronidase and are unable to degrade the glycosaminoglycans (GAGs) dermatan sulfate and heparin sulfate. This results in an accumulation of GAGs within lysosomes, causing multiorgan dysfunction and damage. ${ }^{3,9,15}$ Organs commonly affected include the brain, eyes, ears, nose, throat, heart, lungs, liver, bones, and joints. ${ }^{3}$

A range of symptom severities have been described in association with MPS Type 1 and can be classified into 2 categories: a severe form of MPS Type 1 (Hurler syndrome), and an attenuated form of MPS Type 1 (HurlerScheie and Scheie syndromes). ${ }^{9}$ The severe form of MPS Type 1 accounts for the majority of cases. ${ }^{9}$

Children with Hurler syndrome experience progressive neurocognitive decline due the accumulation of GAGs in the brain. This typically manifests as developmental delay and/or developmental regression between 1 and 2 years of age. ${ }^{9}$ Communicating hydrocephalus is also a common finding in children with Hurler syndrome and can cause additional neurodevelopmental challenges in children who are already neurologically impaired. ${ }^{1,9}$ If untreated, Hurler syndrome commonly leads to premature death within the 1st decade of life..$^{2,5,11}$
A ventriculoperitoneal shunt has often been used to treat individuals with communicating hydrocephalus, typically before hematopoietic stem cell transplant (HSCT). ${ }^{1}$

In this report, we describe a unique case of ventriculomegaly regression following medical management of MPS Type 1 . We explore the possible mechanisms related to the findings in our case and discuss the implications for treatment.

\section{Case Report}

History and Examination

This 3-year-old boy initially presented to British Columbia Children's Hospital at 20 months of age with macrocephaly, coarse facial features, developmental regression, corneal clouding, obstructive sleep apnea, skeletal contractures, and an umbilical hernia. He was born following an uncomplicated pregnancy via cesarean section at 42 weeks due to macrocephaly. His parents are nonconsanguineous, and there was no prenatal teratogen exposure. Clubfoot was diagnosed at birth and was treated with casting. Results of a neonatal hearing screen were found to be nonsatisfactory. At 3 months of age, bilateral inguinal hernias were repaired, and at 11 months of age, bilateral tympanostomy tubes were inserted for multiple serous otitis media. Review of developmental milestones found 
that the patient was sitting unsupported at $7-8$ months and was walking by 15 months. At 20 months, the patient's gait appeared unstable, and frequent falls and trips were reported. Expressive language skills regressed from 1 to 2 words at 1 year of age to no words at the time of initial assessment. Receptive language skills were also impaired, and sensorineural hearing loss was suspected.

At 20 months of age, the patient's head circumference was measured to be $54.5 \mathrm{~cm}$, which corresponds to greater than 2 standard deviations above the mean. Frontal bossing was also noted. No papilledema was found on funduscopic examination. A prominent metopic suture line suggested metopic synostosis. Splenomegaly and a systolic heart murmur were also noted.

A CT head scan showed low attenuation in the periventricular white matter with prominent lateral and third ventricles. This was followed by an MRI scan that showed T2 signal hyperintensity in the periventricular and deep white matter as well as evidence of ventriculomegaly (Fig. 1 left). Given the clinical findings, imaging results, urine mucopolysaccharides of $91 \mathrm{mg} / \mathrm{mmol}$ creatinine (reference level $<22 \mathrm{mg} / \mathrm{mmol}$ creatinine) and $\alpha$-1-iduronidase activity of $8.1 \mathrm{pmol} / \mathrm{punch} / \mathrm{hr}$ on blood spot enzyme testing (reference level $\geq 16.8$ ), the diagnosis of Hurler syndrome was confirmed.

\section{Treatment}

Enzyme replacement therapy (ERT) with laronidase (Aldurazyme) was started immediately. At 25 months of age, this patient received an allogenic umbilical cord HSCT from an unrelated donor.

\section{Posttreatment Course}

Repeat MRI was performed 1 year later at 32 months of age. Findings included a decrease in T2 signal hyperintensity in the periventricular and deep white matter. In addition, ventriculomegaly had improved (Fig. 1 right). Head circumference was $55 \mathrm{~cm}$, keeping him at greater than 2 standard deviations about the mean. Clinically, improvements in developmental motor milestones were seen. He was able to walk with a normal gait pattern, climb stairs unassisted, and feed himself. Progress in speech and language development was also noted. Although he was still nonverbal, he displayed the ability to make more sounds and showed more expression.

\section{Discussion}

Hurler syndrome is the most severe form of MPS and is often associated with progressive neurocognitive decline.$^{5,9,15}$ In this case, ventricular enlargement and periventricular signal change were noted on the initial MR image; however, given that there were no signs to suggest increased intracranial pressure, no surgical CSF diversion was initially completed.

HSCT is currently the gold standard treatment for Hurler syndrome in individuals diagnosed by 2.5 years of age. ${ }^{5}$ ERT can be started prior to grafting to optimize HSCT success, as was done in our case..$^{5}$ On the follow-up MRI, white matter changes were noted, as well as a decrease in ventricular enlargement. Wang et al. ${ }^{14}$ reported on 3
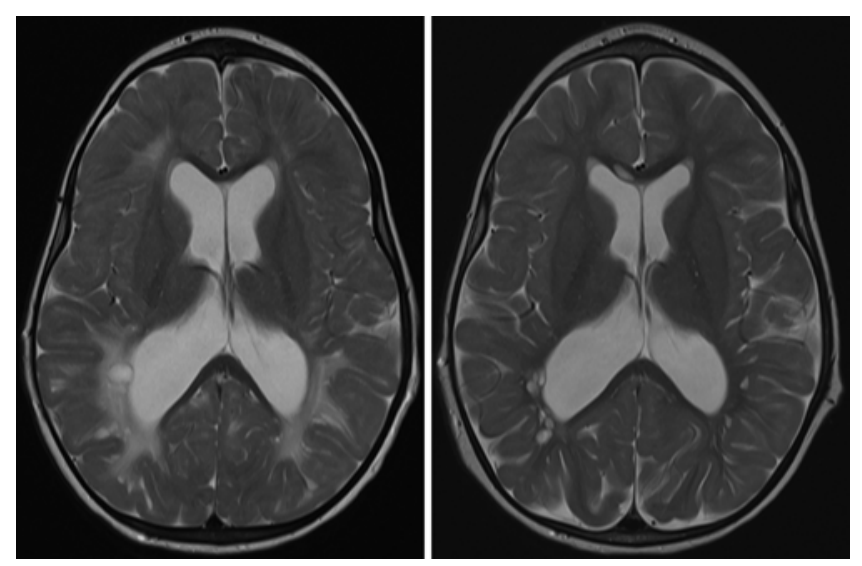

FIG. 1. T2/FLAIR MR images obtained at 20 months of age before starting ERT and before HSCT (left) and at 32 months of age after 12 months of ERT/7 months post-HSCT (right). Signal hyperintensity in the periventricular and deep white matter as well as ventriculomegaly decreased significantly following treatment.

patients with MPS Type 1, where white matter changes improved; one of these patients experienced some posttreatment decrease in ventricular size. That study did not present radiographic documentation of the pretreatment ventriculomegaly or the posttreatment changes that were stated to have occurred in one case within their series.

The reasons for treatment-related improvement in ventriculomegaly are not entirely clear. Meningeal deposition of GAGs has been thought to affect CSF resorption in the arachnoid granulations and may lead to ventriculomegaly and hydrocephalus. ${ }^{1,2,11}$ HSCT may help decrease GAG storage, improve CSF flow, and decrease ventricular size. ${ }^{14}$ In addition, cerebral atrophy as a consequence of MPS affecting the brain parenchyma may contribute to the development of ventriculomegaly. ${ }^{2,6,8}$ Following HSCT, reversal of periventricular changes may have additional effects on ventricular size. ${ }^{4,7,12}$

A limitation to this case is that only one follow-up MRI study was obtained, 1 year after starting ERT and 7 months post-HSCT. Takahashi et al. demonstrated a transient increase in ventricular size in a Hurler patient postHSCT with no overall net change in ventricular size. ${ }^{13}$ Unfortunately, we are unable to comment on how early ventricular size improvement occurred and if there were any transient changes.

Our case contributes to the current understanding of Hurler's syndrome, with radiological improvement in ventriculomegaly as well as improvement in periventricular T2 signal changes. The clinical and radiographic circumstances of Hurler's syndrome patients at the time of neurosurgical referral (such as macrocephaly, ventriculomegaly, or periventricular T2 signal change) might lead many to contemplate surgical CSF diversion. However, the more prudent course of action may well be to await the improvements associated with medical management of the underlying MPS.

\section{References}

1. Aliabadi H, Reynolds R, Powers CJ, Grant G, Fuchs H, Kurtzberg J: Clinical outcome of cerebrospinal fluid shunting 
for communicating hydrocephalus in mucopolysaccharidoses I, II, and III: a retrospective analysis of 13 patients. Neurosurgery 67:1476-1482, 2010

2. Campos D, Monaga M: Mucopolysaccharidosis type I: current knowledge on its pathophysiological mechanisms. Metab Brain Dis 27:121-129, 2012

3. Connock M, Juarez-Garcia A, Frew E, Mans A, Dretzke J, Fry-Smith A, et al: A systematic review of the clinical effectiveness and cost-effectiveness of enzyme replacement therapies for Fabry's disease and mucopolysaccharidosis type 1 . Health Technol Assess 10:iii-iv, ix-113, 2006

4. El-Amouri SS, Dai M, Han JF, Brady RO, Pan D: Normalization and improvement of CNS deficits in mice with Hurler syndrome after long-term peripheral delivery of BBB-targeted iduronidase. Mol Ther 22:2028-2037, 2014

5. Jameson E, Jones S, Wraith JE: Enzyme replacement therapy with laronidase (Aldurazyme ${ }^{\circledR}$ ) for treating mucopolysaccharidosis type I. Cochrane Database Syst Rev 11:CD009354, 2013

6. Lee C, Dineen TE, Brack M, Kirsch JE, Runge VM: The mucopolysaccharidoses: characterization by cranial MR imaging. AJNR Am J Neuroradiol 14:1285-1292, 1993

7. Lücke T, Das AM, Hartmann H, Sykora KW, Donnerstag F, Schmid-Ott G, et al: Developmental outcome in five children with Hurler syndrome after stem cell transplantation: a pilot study. Dev Med Child Neurol 49:693-696, 2007

8. Matheus MG, Castillo M, Smith JK, Armao D, Towle D, Muenzer J: Brain MRI findings in patients with mucopolysaccharidosis types I and II and mild clinical presentation. Neuroradiology 46:666-672, 2004

9. Muenzer J, Wraith JE, Clarke LA: Mucopolysaccharidosis I: management and treatment guidelines. Pediatrics 123:1929, 2009

10. Neufeld E, Muenzer J: The mucopolysaccharidoses, in Scriver CR, Beaudet AL, Sly WS, et al (eds): The Metabolic and Molecular Bases of Inherited Disease. New York: McGraw-Hill, 2001, pp 3421-3452

11. Sheridan M, Johnston I: Hydrocephalus and pseudotumour cerebri in the mucopolysaccharidoses. Childs Nerv Syst 10:148-150, 1994
12. Souillet G, Guffon N, Maire I, Pujol M, Taylor P, Sevin F, et al: Outcome of 27 patients with Hurler's syndrome transplanted from either related or unrelated haematopoietic stem cell sources. Bone Marrow Transplant 31:1105-1117, 2003

13. Takahashi Y, Sukegawa K, Aoki M, Ito A, Suzuki K, Sakaguchi $\mathrm{H}$, et al: Evaluation of accumulated mucopolysaccharides in the brain of patients with mucopolysaccharidoses by ${ }^{1} \mathrm{H}$-magnetic resonance spectroscopy before and after bone marrow transplantation. Pediatr Res 49:349-355, 2001

14. Wang RY, Cambray-Forker EJ, Ohanian K, Karlin DS, Covault KK, Schwartz PH, et al: Treatment reduces or stabilizes brain imaging abnormalities in patients with MPS I and II. Mol Genet Metab 98:406-411, 2009

15. Wraith JE, Clarke LA, Beck M, Kolodny EH, Pastores GM, Muenzer J, et al: Enzyme replacement therapy for mucopolysaccharidosis I: a randomized, double-blinded, placebocontrolled, multinational study of recombinant human $\alpha$-Liduronidase (laronidase). J Pediatr 144:581-588, 2004

\section{Disclosures}

The authors report no conflict of interest concerning the materials or methods used in this study or the findings specified in this paper.

\section{Author Contributions}

Conception and design: both authors. Acquisition of data: Liang. Analysis and interpretation of data: both authors. Drafting the article: both authors. Critically revising the article: both authors. Reviewed submitted version of manuscript: both authors. Approved the final version of the manuscript on behalf of both authors: Singhal.

\section{Correspondence}

Ash Singhal, British Columbia's Children's Hospital, 4480 Oak St., Rm. K3-216, Vancouver, BC V6H 3V4, Canada. email: asinghal@cw.bc.ca. 